\title{
Obtenção de um diagrama pseudoternário utilizando tween 80 como tensoativo
}

Gabrielly de Lucena Tiburtino*; (Graduanda em Engenharia Mecânica na Universidade Federal Rural do Semiárido-UFERSA)

Maria Aparecida Bezarra Oliveira; (Graduanda em Engenharia Civil na Universidade Federal Rural do Semiárido -UFERSA)

Adna Lúcia Rodriguez Menezes; (Graduanda em Engenharia Civil na Universidade Federal Rural do Semi-árido -UFERSA)

Guymmann Clay da Silva. (Dra. Professora Química na Universidade Federal Rural do Semi-árido - UFERSA)

*Email:gabriellyy_lucena@hotmail.com

\section{Resumo:}

Os tensoativos não iônicos são amplamente utilizados em diferentes campos. Uma das características mais interessantes destes tensoativos é a sua capacidade de formar sistemas micelares estáveis. Neste trabalho, foram obtidos dois diagramas pseudoternário, através da mistura em tubos de ensaio do tensoativo Tween 80 , cotensoativo $\mathrm{N}$-butanol em mesmas proporções, logo após adicionando-se a fase óleo que variou ( $\mathrm{N}$ - hexano e xileno) até atingir a razão $\mathrm{C} / \mathrm{T}=1$ e por fim acrescentou-se a fase aquosa gota a gota até que se obtivesse a emulsão de aparecia turva para que assim fosse determinada as regiões de emulsão e microemulsão que foram plotadas em gráficos. Dessa forma, foi feito o estudo da variação da fase óleo. Os resultados mostram que a variação da fase óleo escolhida não apresentou mudança na região de microemulsão. Isso foi atribuído ao fato de que o hexano pode migrar para a fase óleo, por ser apolar, ou o mesmo ter evaporado antes de microemulsionar. Em relação ao diagrama obtido com o xileno, a literatura diz que o hidrocarboneto com anéis benzênicos diminui a região de microemulsão. Com os resultados obtidos, podem ser realizados testes de viscosimetria capilar, densidade e medidas de turbidez. Esses sistemas obtidos são muito estáveis e de fácil preparo podendo ser utilizados, não apenas em tratamento de efluentes para remoção de petróleo, mas para uma vasta aplicação na indústria, como reportado na literatura.

\section{Palavras-chave:}

Micelas; Microemulsão; Eflentes. 


\section{I ntrodução}

As microemulsões são definidas como sistemas de alta agregação nos qual água e óleo são homogeneamente misturados devido à presença de substâncias anfifílicas chamadas de tensoativos. Os tensoativos possuem em sua cadeia uma parte polar, capaz de se misturar a substâncias polares e outra apolar capaz de se misturar a compostos apolares ou orgânicos. Esses tensoativos quando adicionados a um meio aquoso ou oleoso tendem a formar, acima da CMC (concentração micelar crítica), pequenos aglomerados coloidais chamados de micelas. Essas micelas podem ser do tipo direta, ou seja, óleo/água (O/A), ou do tipo inversa, água/óleo (A/O). Essas estruturas internas das micelas (geometrias) existentes em microemulsão podem ser variadas, complexas e podem ser físico-quimicamente entendidas.

Dependendo das concentrações dos constituintes da microemulsão uma grande diversidade de estruturas entre a fase contínua e dispersa pode ser identificada (SILVA, 2011), proporcionando maior solubilização do óleo presente na água. Suas propriedades os tornam sistemas altamente versáteis e de interesse em diversos domínios de aplicação (GOMES, 2009). Gomes (2009) e Siroky (2010), estudaram diferentes sistemas microemulsionados que possibilitam uma maior solubilização da fração pesada do óleo (borra).

Diante da necessidade de aperfeiçoar técnicas que sejam eficazes na remoção de óleo presente no efluente, precisa-se de um sistema estável, de fácil preparo, espontâneo, e que facilite a solubilização de diversos compostos. Um sistema muito utilizado em diversas áreas da indústria devido suas características típicas, são os sistemas microemulsionados (VIANA, 2010). Suas propriedades e seu grande poder de solubilização os tornam excelentes solventes para contaminantes polares e apolares, ampliando em muito as vantagens de aplicação de tensoativos em solução (SIROKY, 2010). Esses sistemas, sejam eles sistemas micelares diretos ou inversos, já são aplicados em diversos campos industriais (STICKDORNT, 1995) tais como na indústria farmacêutica (STICKDORNT, 1995; ESPOSITO, 2003; FENG-FENG, 2005; FANUN, 2008) e de cosméticos (STICKDORNT, 1995; FANUN, 2008), na indústria têxtil (STICKDORNT, 1995), na indústria de petróleo (SCHULZ, 2005), dentre outros. As microemulsões diferem das emulsões não apenas por seu tamanho estrutural ser muito menor, tamanho nanométrico, mas também na sua estabilidade termodinâmica, estabilizando e proporcionando vida longa a sistemas de misturas do tipo óleo/água ou água/óleo (SILVA, 2011).

Considerando que de todas as crises sociais e naturais que os seres humanos devem enfrentar, a dos recursos hídricos é a que mais afeta a nossa sobrevivência. Admitindo que o consumo mundial aumentou 35 vezes ao longo dos três últimos séculos, em função do aumento do consumo em residências, indústrias, dentre outras, a água tem sido utilizada em quantidades superiores ao volume disponível, gerando problemas de escassez (SCHULZ, 2005).

O processo de racionalização da água é uma questão que vem ganhando importância nos últimos anos, uma vez que este recurso está se tornando cada vez mais escasso (TOZE, 2006). O problema da escassez da água é devido ao seu mau uso nos últimos anos, tanto pela sociedade, quanto pelo setor industrial, que a utiliza em todas as suas atividades, produzindo grandes quantidades de efluentes (GOGATE, 2004). Os contaminantes presentes nestes efluentes industriais são os mais diversos possíveis: corantes, sólidos suspensos, metais pesados, matéria orgânica, nutrientes, nitratos, taninos, ligninas, princípios ativos, dentre outros (ATTIA, 2006).

No caso da indústria de petróleo, em decorrência de uma intensa atividade de exploração e produção de petróleo, esta, é responsável por grande parte da geração de resíduos (GUIMARÃES, 2007), uma vez que a exploração e o refino de petróleo são algumas das mais importantes atividades industriais da sociedade moderna, e seus derivados são empregados em vários destinos (SCHULZ, 2005) os quais são considerados tóxicos e poluentes ao meio ambiente (GUIMARÃES, 2007).

Dentre estes resíduos, pode-se citar: os resíduos oleosos e viscosos formados durante as etapas de produção; resíduos formados a partir do transporte de refino de petróleo; de filtros de petróleo nas plataformas; tanques de navios petroleiros; oleodutos; da limpeza de permutadores e da parte inferior dos tanques de armazenamento de petróleo e seus derivados; como: diesel; gasolina; GLP; nafta petroquímica; solventes como querosene; óleos lubrificantes; parafinas óleo combustível; asfalto dentre outros (GUIMARÃES, 2007). Neste caso, o principal contaminante na indústria de petróleo, que é uma grande consumidora de água, são os resíduos de óleo (SANTO, 2012), gerando assim grandes quantidades de efluentes (SCHULZ, 2005) como citados anteriormente. Neste caso a água é utilizada 
para diversos fins como: produção de vapor d'água, água de processo, proteção contra incêndio, uso doméstico, dentre outra (DIEPOLDER, 1992; SCHULZ, 2005).

Devido ao forte impacto que causam ao meio ambiente, esses efluentes são rigorosamente controlados pelas Legislações Ambientais vigentes, o que faz com que a indústria busque cada vez mais alternativas econômicas e ecologicamente viáveis para seu tratamento. Devido a isso, vários estudos sobre tecnologias foram desenvolvidas para o tratamento de efluentes industriais. A seleção da tecnologia depende das características do efluente e da qualidade que se deseja para o produto final, além de custo e facilidade de operação do processo (SCHULZ, 2005).

Nas refinarias de petróleo, o tratamento de efluentes está dividido em quatro níveis: tratamento primário, que envolve processos físicos de tratamento; tratamento secundário, que compreende as operações onde a matéria solúvel é removida; e tratamentos terciário e quaternário que seriam o polimento final do efluente, para descartá-lo dentro dos padrões exigidos pela Legislação vigente. Desconsiderando os níveis de tratamento, as operações para tratamento de efluentes são classificadas em físicas (flotação, coalescência, filtração), químicas (precipitação, coagulação, troca iônica), térmicas e biológicas (BEGAJEWICZ, 2000).

Para Gryta et al. (2001), os métodos mecânicos para tratamento de efluentes oleosos são baseados no fenômeno de quebra da emulsão através da gravidade. Para os mesmos autores os métodos químicos são primeiramente baseados na neutralização de detergentes (estabilizantes de emulsões) e na mudança de $\mathrm{pH}$ da solução. Isto resulta na aceleração do processo de separação devido ao efeito da coalescência. A fase aquosa do tratamento químico contém óleo residual, entretanto, este deve ser submetido a posterior purificação para promover efluente com características de efluente doméstico;

- $\quad$ Para Srijaroonat et al. (1999), várias técnicas tradicionais são usadas para tratamento do efluente oleoso. O óleo livre pode ser facilmente separado por meios mecânicos, como sedimentação por gravidade (separador API “American Petroleum Institute), "skimming”, flotação por ar dissolvido (DAF "Dissolved Air Flotation”), coalescência e centrifugação. Para emulsões instáveis que contêm gotas de óleo com diâmetro maior que $100 \mu \mathrm{m}$, técnicas de separação química como floculação e coagulação são aplicadas. Mas todas as técnicas descritas acima têm limitações para separar gotas com diâmetros menores que $20 \mu \mathrm{m}$. Devido à sua estabilidade, algumas destas são impossíveis de quebrar por meios químicos;

Para Gryta et al. (2001), os métodos tradicionais de tratamento de efluentes oleosos não são eficientes especialmente quando as gotas de óleo são finamente dispersas e sua concentração é muito baixa. Segundo os autores existe ainda a necessidade de um método universal, que seja eficiente em todos os casos, para remoção de óleo em efluentes.

Os métodos tradicionais de tratamentos de efluente de petróleo são baseados em métodos físicos e químicos. De acordo com os autores citados anteriormente, Srijaroonat et al. (1999), esses métodos em algumas situações específicas como tamanho de gotas menores que 20 micrômetros, por exemplo, não são eficazes devido à instabilidade do sistema. Para Gryta et al. (2001) ainda a necessidade de um método universal, que seja eficiente em todos os casos, para remoção de óleo de efluentes. Logo, há uma necessidade de otimizar técnicas, que sejam eficazes na remoção de óleo presente no efluente. Essa técnica ou sistema precisa ser estável, de fácil preparo, espontâneo, e que facilite a solubilização de diversos compostos. O estudo destes sistemas será efeito para dois tipos de fase óleo separadamente, sendo primeiro o hexano e depois o xileno, utilizando Tween 80 como tensoativo, N-butanol como cotensoativo e fase aquosa, a uma razão $\mathrm{C} / \mathrm{T}=1,0$. O motivo de utilizar o tensoativo não iônico é que ele vai proporcionar maior estabilidade à microemulsão. O cotensoativo que vai ser utilizado é um álcool de cadeia curta não ramificada (butanol), que além de proporcionar maior estabilidade a microemulsão aumentando o poder de solubilização da mesma.

Portanto, o objetivo deste trabalho é obter diagramas pseudoternário utilizando um tensoativo não iônico, um cotensoativo (álcool), fase óleo (xileno e N-hexano) e água destilada como fase aquosa, que possa ser utilizado para tratamento de efluente de indústria de petróleo.

\section{Metodologia}


Para se realizar o processo de microemulsão foi necessário que fosse pesado as massas de cada componente da solução e para isso foi usada uma balança analítica, após esse processo se fez necessário um agitador mecânico para que se misturasse a solução composta por tensoativo Tween 80 , cotensoativo o álcool butílico, as fases óleo hexano e o xileno e a água destilada (Figura 1).

\subsection{Materias e reagentes}

\begin{tabular}{|c|c|c|c|c|c|c|c|}
\hline & $\begin{array}{l}\text { Balança } \\
\text { analítica }\end{array}$ & $\begin{array}{l}\text { Agitador } \\
\text { mecânico }\end{array}$ & $\begin{array}{c}\text { Tween } \\
80\end{array}$ & $\begin{array}{l}\text { Álcool } \\
\text { butílico }\end{array}$ & Hexano & Xileno & Agua \\
\hline INFOMAÇÕES & $\begin{array}{l}\text { SHIMADZU, } \\
\text { modelo: } \\
\text { AUY220, } \\
\text { Máx }=220 \mathrm{~g}, \\
\text { Mín }=10 \mathrm{mg}, \\
\mathrm{e}=1 \mathrm{mg} \\
\mathrm{d}=0,1 \mathrm{mg}\end{array}$ & $\begin{array}{l}\text { Norte } \\
\text { científica } \\
\text { NA } \\
3600 \\
\text { modelo: } \\
\text { agitador } \\
\text { de } \\
\text { amostras. }\end{array}$ & $\begin{array}{l}\text { 110/220, } \\
\text { POT: } \\
130 \mathrm{~W} \\
(\mathrm{CRQ})^{\mathrm{R}}\end{array}$ & $\begin{array}{l}\text { PRQ } \\
\text { químicos }\end{array}$ & $\begin{array}{l}\text { P.A., } \\
\text { Synth }\end{array}$ & $\begin{array}{l}\text { P.A., } \\
\text { Synth }\end{array}$ & Destilada \\
\hline
\end{tabular}

Figura 1: Informações dos materiais e reagentes usados/Fonte:autor

\subsection{Obtenção do diagrama pseudoternário}

Os sistemas de dispersão foram preparados usando a metodologia de titulação da fração mássica utilizou uma balança analítica e agitadores mecânicos, à temperatura de $27^{\circ} \mathrm{C} \pm 0,1^{\circ} \mathrm{C}$, conforme descrito em trabalhos pesquisados (DANTAS NETO, 1993; WANDERLEY NETO, 2010). Após a determinação da razão C/T, fez-se o cálculo da massa de contesoativo, tensoativo e fase óleo que foram usados em mesma proporção.

Uma dada massa de tensoativo, cotensoativo e fase óleo foram adicionadas a um tubo de ensaio. Gota a gota, água foi adicionada ao sistema e levada a um agitador mecânico. Esse procedimento de adição de água terminou quando o processo atingiu a fase de emulsão, que foi percebida no momento em que o sistema turvou, mudando sua coloração de incolor para turvo. Após esse procedimento a solução foi pesada para se obter o peso da fase aquosa que foi adicionada. Com o peso de cada constituinte obtido cálculos foram feitos para se obter a porcentagem de massa de cada substância na fase de microemulsão. Os cálculos foram feitos pela razão entre a massa de cada substância, tensoativo, cotensoativo, fase óleo e fase aquosa, pela massa de todo o sistema, como mostram as Equações 1-4.

$$
\begin{aligned}
& \% m_{T}=\frac{m_{T}}{m_{T S}} \times 100 \% \\
& \% m_{C T}=\frac{m_{C T}}{m_{T S}} \times 100 \% \\
& \% m_{F o}=\frac{m_{F o}}{m_{T S}} \times 100 \% \\
& \% m_{A}=\frac{m_{A}}{m_{T S}} \times 100 \%
\end{aligned}
$$

onde, $m_{T S}$ massa total do sistema; $m_{T}$ massa de tensoativo; $m_{C T}$ massa de cotensoativo; $m_{F o}$ massa de fase óleo; $m_{A}$ massa de água.

\subsection{Obtenção das extensões das regiões microemulsionadas}


Com os resultados da percentagem de massa de cada componente foram plotados no Origin para se obter os diagramas pseudoternários, esses foram imprimidos em folhas de papel, cortados e pesados em uma balança analítica digital. Através do peso da região de microemulsão, também pesada, foi calculada a porcentagem de acordo com a Equação 5, como reportado na literatura (MORAIS, 2009).

$$
\% R M=(P R M / P D) * 100
$$

onde, $\% R M=$ percentagem da região microemulsionada, $P R M=$ peso da região microemulsionada e $P D=$ peso total do diagrama.

\section{Resultados e discussão}

\subsection{Obtenção dos diagramas pseudoternários}

A obtenção de sistemas pseudoternários requer o uso de um cotensoativo, molécula que possibilita o aumento da solubilidade do sistema e pode, também aumentar a estabilidade do sistema, devido agir na interface das duas fases (MORAIS, 2009). A escolha do tensoativo Tween 80 foi feita devido o mesmo ser um tensoativo não-iônico. Esse tipo de tensoativo estabiliza melhor a microemulsão, uma vez que a interação entre as cabeças do tensoativo na micela são menores, por não ter íons (Figura 2), do que os tensoativos iônicos.

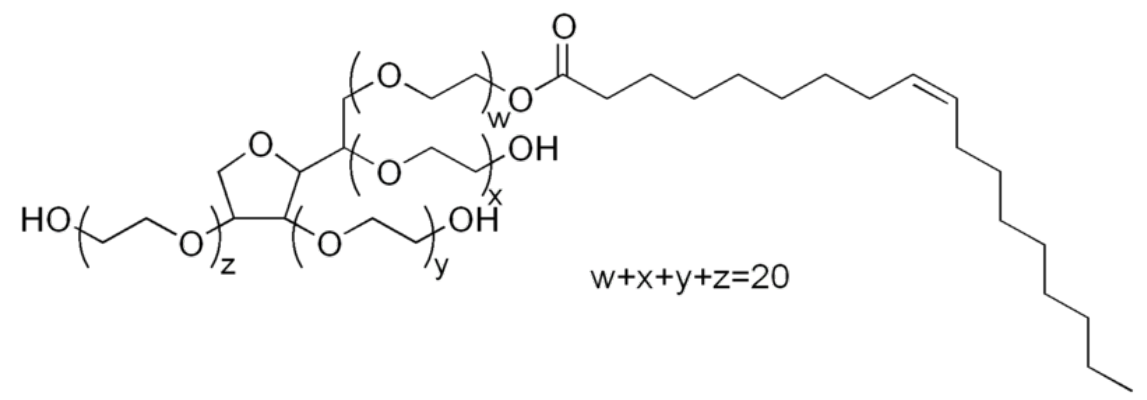

Figura 2: Fórmula molecular do Tween 80 (Polisorbato)

Alguns parâmetros que influenciam na microemulsão são a influência do cotensoativo, a isomeria do álcool, a influência da razão cotensoativo/tensoativo, estrutura do hidrocarboneto, a influência da salinidade, e a influência do pH (SOUZA, 2006). No presente trabalho, foram obtidos sistemas contendo tensoativo não iônico, cotensoativo de cadeia curta, com razão $C / T=1,0$, objetivando estudar a influência da fase óleo, como reportado na literatura (DANTAS T.N.C. et. al., 2009). Na Figura 3, foi utilizado o xileno, e na Figura 4 foi utilizado o hexano como fase óleo. Como pode ser visto, a proposta de estudar os diagramas pseudoternário, utilizando o mesmo tensoativo e cotensoativo a uma razão $\mathrm{C} / \mathrm{T}=1$, variando apenas a fase óleo não foi bem-sucedida, uma vez que a região de microemulsão para a ambos os diagramas são praticamente iguais.

O xileno é uma mistura de seus isômeros comuns (orto, meta e para) (DANTAS T.N.C. et. al., 2009) e o hexano é um hidrocarboneto alifático. A substituição do hidrocarboneto alifático pelo aromático induz fortes interações entre tensoativo e óleo, provocando a rigidez do filme interfacial e diminuindo a região de microemulsão, ponto IV nos diagramas. A estrutura da fase óleo, Fo, influência nas propriedades da interface, de acordo com modelos propostos por Winsor (WINSOR, 1954) em 1954. Efeitos intensos de solvatação nas interfaces do sistema são promovidos pelas interações tensoativoóleo em função da natureza variável das moléculas de óleo (pequeno volume molecular nos hidrocarbonetos de cadeia curta até alta polaridade com o aumento da aromaticidade). Por outro lado, o aumento no comprimento da cadeia do óleo conduz a uma redução das interações entre as microgotículas, diminuindo a capacidade de solubilização da microemulsão (LEUNG, R.; SHAH, D. O.J ., 1986). Em processos de separação, essa propriedade é fundamental. 
A Figura 3 mostra o digrama pseudoternário obtidos com o Tween 80, como tensoativo, butanol como cotensoativo, xileno como fase óleo e água, com razão $\mathrm{C} / \mathrm{T}=1,0$.

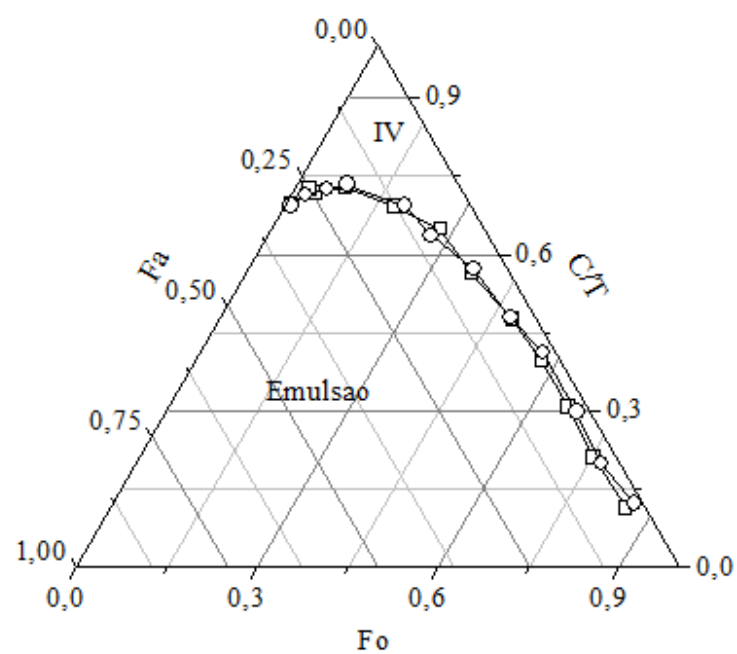

Figura 3: Diagrama pseudoternário, utilizando Tween 80, butanol, xileno e água.

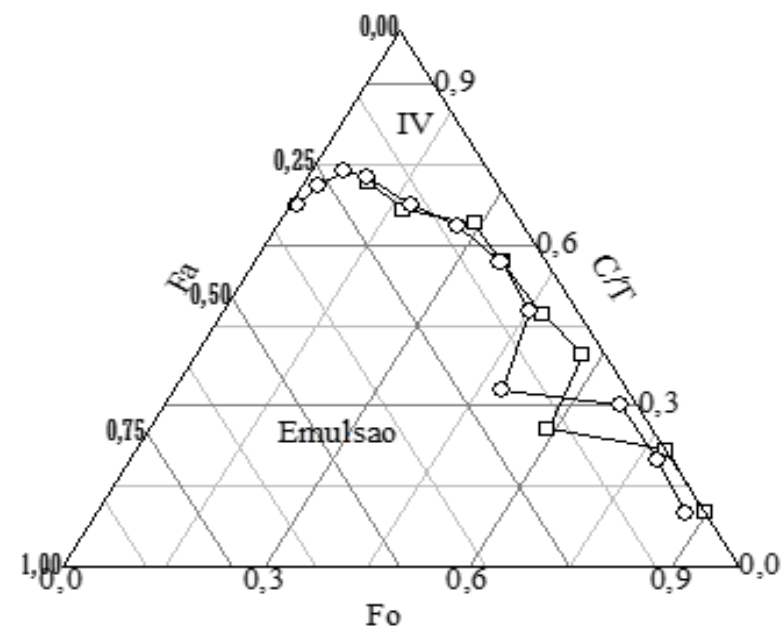

Figura 4: Diagrama pseudoternário, utilizando Tween 80, butanol, hexano e água.

De acordo com a literatura a substituição do hidrocarboneto alifático (Figura 4) pelo aromático (Figura 3) induz a diminuição da região de microemulsão. Os cálculos da \% RM para o diagrama da Figura 3 é 14,44 \% e 17,97 \% para o diagrama da Figura . Apesar da \% RM ser maior para o diagrama com a fase óleo alifática, (hexano), esperava-se que essa região de microemulsão fosse muito maior, pois a diferença de \% RM é apenas 3,53 \%. Isso pode ser explicado por dois mecanismos propostos:

1. O hexano é um composto alifático, basicamente apolar e insolúvel na fase aquosa. Devido a essas características, o hexano migra facilmente da fase contínua, fase aquosa, para a fase dispersa, fase oleosa, aumentando o tamanho da micela e consequentemente aumentando a região de emulsão, como mostra o esquema da Figura 4. 


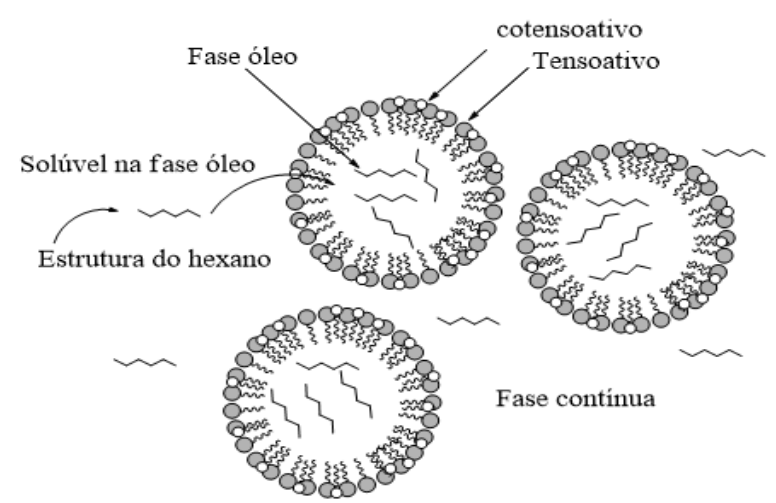

Figura 3: Esquema de migração do hexano da fase contínua, para a fase dispersa.

A diferença entre a emulsão e microemulsão, não está apenas na estabilidade termodinâmica, mas também no tamanho estrutura da micela (LIF and HOLMBERG, 2006). A região caracterizada como IV nos diagramas é a região de microemulsão, região microscopicamente homogênea composta de uma única fase, que no caso é a microemulsão (DANTAS T.N.C. et. al., 2009). A outra região, é a de emulsão, região que apresenta tamanho de micelas maiores e instáveis (LIF and HOLMBERG, 2006). Essa caracterização foi obtida a partir da classificação das regiões de Winsor.

O hexano é um solvente muito volátil, ou seja, antes de microemulsionar parte do hexano evapora, motivo esse que apresenta uma variação nos pontos em equilíbrio da emulsão com a microemulsão.

Nesses pontos, a quantidade de tensoativo e contesoativo é muito baixa $(0,2-0,5)$, ou seja, antes do hexano microemulsionar, migrar para dentro da micela, o mesmo evapora, comprometendo a região de microemulssão.

\section{Conclusões}

De acordo com os resultados obtidos é possível concluir que:

- Através da titulação mássica com a fase aquosa, foi possível a determinação de regiões de microemulsão em diagramas pseudoternários, utilizando tensoativo não-iônico, Tween 80;

- A estrutura dos tensoativo Tween 80 não contribuiu para uma diferença no tamanho das regiões de Winsor IV em ambos os diagramas;

- A utilização de uma fase óleo volátil pode mascarar ou comprometer a região de microemulsão;

- Cálculos da \% RM mostram que os hidrocarbonetos alifáticos proporcionam uma região de microemulsão maior, com 17,97 \%, do que os hidrocarbonetos aromáticos, com 14,44 \%;

- A região de microemulsão é mais dependente da estrutura do tensoativo, do cotensoativo e da razão C/T do que da estrutura da fase óleo;

- De acordo com os diagramas obtidos, trabalhos futuros podem serem feitos, variando a razão $\mathrm{C} / \mathrm{T}$, variando o tensoativo e variando o cotensoativo;

Figura 4: Diagrama pseudoternário, utilizando Tween 80, butanol, hexano e água.

- Dentro das regiões de microemulsão pode ser obtido diferentes pontos de microemulsão que futuramente serão aplicados na indústria petrolífera para a remoção de óleo em efluentes, solubilização de borra, recuperação avançada dentre outras podendo também ser utilizado em processos que não estejam ligados ao petróleo.

\section{Agradecimentos}

Guymmann Clay da Silva pela orientação. Wilsdon Arcanjo de Morais pelo auxilio laboratorial. UFERSA; UFRN; UERN. CNPq. 


\title{
Obtaining a pseudo ternary diagram using tween 80 as surfactant
}

\begin{abstract}
The nonionic surfactants are widely used in different fields. One of the most interesting features of these surfactants is their ability to form stable micellar systems. In this study, two pseudoternário diagrams were obtained by mixing in surfactant vials Tween 80 , cosurfactant $\mathrm{N}$-butanol in the same proportions, after adding the oil phase ranging ( $\mathrm{N}$ - hexane and xylene) until the ratio $\mathrm{C} /$ $\mathrm{T}=1$ and finally added to the aqueous layer drop by drop until achieving the appeared cloudy emulsion was determined so that the regions of emulsion and microemulsion were plotted on graphsThus, the study of variation of the oil phase was carried out. The results show that the variation of the selected oil phase showed no change in the region of microemulsion which was attributed to the fact that the hexane can migrate to the oil phase, as it is polar, or even it has evaporated before it microemulsifies. In relation to the diagram obtained with xylene, the literature says that the hydrocarbon with the benzene rings lowers the microemulsion region. With the obtained results, capillary viscometry, and density measurements of turbidity tests can be carried out. These obtained systems are very stable and easy to prepare and can be used not only in wastewater treatment for removal of oil, but for a wide application in industry as reported in the literature.
\end{abstract}

Keywords:Micelles; Microemulsion; Effluents.

\section{Referências bibliográficas}

ATTIA, A. A. R., W. E.; KHEDR, S. A. " Capacity of activated carbon in the removal acid dyes subsequent to its thermal treatment." Dyes Pigments 69: 128-136, 2006.

BEGAJ EWICZ, M. "A review of recent design procedures for water networks in refineries and process plants."Computers and Chemical Engineering, 24: 2093-2113, 2000.

DANTAS NETO, A. A.; DANTAS T. N. C.; DUARTE, M. M. L.; AVELINO, S. "Equilibrium diagrams at 27 [degree]C of the water + sodium tungstate + dodecylamine chloride system." Journal of Chemical and Engineering, 38,: 67-69, 1993.

DANTAS, Tereza Neuma Castro; Oliveira, K. R. et al. "The use of microemulsions to remove chromium from industrial sludge."Water Research 43(5): 1464-1470, 2009.

DIEPOLDER, R."Is Zero Discharge Realistic?" Hydrocarbon Procesing, 71: 129-131, 1992.

ESPOSITO, E. "Amphiphilic association systems for Amphotericin B delivery." International Lournal of Pharmaceutics 260, 2003.

FANUN, M. " A study of the properties of mixed nonionic surfactants microemulsions by NMR, SAXS, viscosity and conductivity."Journal of Molecular Liquids 142: 1-3, 2008.

FENG-FENG, L. V. P. "Phase behavior of the microemulsions and the stability of the chloramphenicol in the microemulsion-based ocular drug delivery system."International Lournal of Pharmaceutics, 301: 237-246, 2005.

GOGATE, P. R. P. "A review of imperative technologies for wastewater treatment I : oxidation technologies at ambient condition."Adv. Environ. Res, 2004.

GOMES, D. A. A. Aplicação de microemulsões na solubilizações de frações pesadas de petróleo.Departamento de Engenharia Química., Universidade Federal do Rio Grande do Norte: 90, 2009. 
GRYTA, M., KARAKUSLKI, K., MORAWSKI, A. W. "Purification of oily watewater by hibrid UF/ MD."Water Research, 35: 3665-3669, 2001.

GUIMARÃES, A. K. V. Extração do óleo e caracterização dos resíduos da borra de petróleo para fins de reuso. Depatamento de Engenharia Química. Natal, Universidade Federal do Rio Grande do Norte: 157, 2007.

LEUNG, R.; SHAH, D. O.J. Coll. and Inter. Sci.: 120, 330, 1986.

LIF, A. and K. HOLMBERG (2006). "Water-in-diesel emulsions and related systems." Advances in Colloid and Interface Science 123â€"126(0): 231-239, 1986.

MORAIS, W. A. Determinação microemulsionadas utilizando tensoativos não iônicos. Departamento de química. Natal, Universidade federal do Rio Grande do Norte: 31, 2009.

OLIVEIRA, A. G.; SCARPA, M. V.; CORREA, M. A.; CERA, L. F. R.; FORMARIZ, T. P. "Microemulsões: estrutura e aplicações como sistema de liberação de fármacos."Quim. Nova 27: 131-138, 2004.

SANTO, C. E. V.; BOTELO, C. M. S.; AMIT, B.; KUMAR, E.; BOAVENTURA, R. A. R. "Optimization of coagulation "flocculation and flotation parameters for the treatment of a petroleum refinery effluent from a Portuguese plant."Chemical Engineering Journal 130: 117-123, 2012.

SCHULZ, C. K. Tratamento de efluentes oleosos utilizando processos de separação por membranas.Departamento de Engenharia Química. Rio de J aneiro Universidade Federal do Rio de Janeiro: 164, 2005.

SILVA, G. C. Sistema microemulsionado: caracterização e aplicação na indústria de petróleo.Depqrtamento de química. Natal, Universidade Federal do Rio Grande do Norte. Doutora: 157, 2011.

SIROKY, A. N. "Aplicação de planejamento de experimentos nos estudos de sistemas microemulsionados visando à solubilização da fração pesada de petróleo." Simpósio nacional de probabilidade e estatística 19, 2010.

SOUZA, P. F. D. Novas alternativas na quebra de emulsão de petróleo: sistemas microemulsionados.Programa de Pós-Graduação em Engenharia Química, Natal - Universidade Federal do Rio Grande do Norte, 2006.

STICKDORNT, M. J. S. K. "Microemulsions in Technical Processes."Chem. Rev 95: 849-864, 1995.

TOZE, S. "Reuse of effluent water-benefits and risks."Agricultural Water Management, 2006.

VIANA, F.F.; MACEDO, A.M.F.; NETO, A.A.D.; Dantas, T.N.C.; ROSSI, C.G.F.T. " Estudo da Solubilização da borra de petróleo em função do percentual de C/ T."Anais: REUNIÃO ANUAL DA SBPC 62, 2010.

WANDERLEY NETO, A.O.; DANTAS, T. N.C.; NETO, A. A. D.; FONSECA, J. L. C. " Characterization of the Liquid-Gas Interface of Aqueous Systems Containing a Derivative from Castor Oil."Lournal of dispersion science and technology 31: 980-985, 2010. 